\title{
Potential of Essential Oils from Active Packaging to Highly Reduce Ethylene Biosynthesis in Broccoli and Apples
}

Alejandra Navarro-Martínez ${ }^{1}$, Antonio López-Gómez ${ }^{2 *}$ and Ginés Benito Martínez-Hernández ${ }^{1 *}$

1 Food Safety and Refrigeration Engineering Group, Department of Agricultural Engineering, Universidad Politécnica de Cartagena, Paseo Alfonso XIII 48, 30203 Cartagena, Murcia, Spain.

2 Biotechnological Processes Technology and Engineering Lab, Instituto de Biotecnología Vegetal, Universidad Politécnica de Cartagena, Edif I+D+I, Campus Muralla del Mar, 30202 Cartagena, Murcia, Spain

*Correspondence: GinesBenito.Martinez@upct.es; antonio.lopez@upct.es; Tel.: +34968325516

Supplementary data. Major volatile compounds (analysed by gas chromatography as described in [1]) of oregano and cinnamon essential oils.

\begin{tabular}{lcclcc}
\hline \multicolumn{1}{c}{ Oregano } & \multicolumn{2}{c}{ Cinnamon } \\
Compound & $\%$ & & & Compound & $\%$ \\
\hline Carvacrol & 46.11 & & & E-cinnamaldehyde & 74.78 \\
Linalool & 28.02 & & & Eugenol & 3.96 \\
Paracymene & 6.17 & & & Benzaldehyde & 1.02 \\
$\gamma$-terpinene & 5.19 & & & $\alpha$-copaene & 0.33 \\
Myrcene & 2.21 & & & $\beta$-caryophyllene & 0.08 \\
$\alpha$-pinene & 2.08 & & Limonene & 0.05 \\
$\alpha$-terpinene & 1.49 & & Acide benzoique & 0.05 \\
$\beta$-caryophyllene & 1.24 & & $\alpha$-humulene & 0.03 \\
Thymol & 0.99 & & $\beta$-phellandrene & 0.02 \\
Borneol & 0.63 & & $\alpha$-cubenene & 0.02 \\
Terpin-4-ol & 0.58 & & & \\
\hline
\end{tabular}

